\title{
Evaluation of Toll-like, chemokine, and integrin receptors on monocytes and neutrophils from peripheral blood of septic patients and their correlation with clinical outcomes
}

\author{
S.C. Silva ${ }^{1}$, G.L. Baggio-Zappia ${ }^{1}$, M.K.C. Brunialti ${ }^{1}$, M.S.C. Assuncao ${ }^{2}$, L.C.P. Azevedo ${ }^{3}$ \\ F.R. Machado ${ }^{4}$ and R. Salomao ${ }^{1}$ \\ ${ }^{1}$ Disciplina de Infectologia, Departamento de Medicina, Hospital São Paulo, Escola Paulista de Medicina, \\ Universidade Federal de São Paulo, São Paulo, SP, Brasil \\ ${ }^{2}$ Unidade de Terapia Intensiva, Hospital Israelita Albert Einstein, São Paulo, SP, Brasi \\ ${ }^{3}$ Unidade de Terapia Intensiva, Hospital Sírio Libanês, São Paulo, SP, Brasil \\ ${ }^{4}$ Disciplina de Anestesiologia, Departamento de Cirurgia, Hospital São Paulo, Escola Paulista de Medicina, \\ Universidade Federal de São Paulo, São Paulo, SP, Brasil
}

\begin{abstract}
Recognition of pathogens is performed by specific receptors in cells of the innate immune system, which may undergo modulation during the continuum of clinical manifestations of sepsis. Monocytes and neutrophils play a key role in host defense by sensing and destroying microorganisms. This study aimed to evaluate the expression of CD14 receptors on monocytes; CD66b and CXCR2 receptors on neutrophils; and TLR2, TLR4, TLR5, TLR9, and CD11b receptors on both cell types of septic patients. Seventy-seven septic patients (SP) and 40 healthy volunteers (HV) were included in the study, and blood samples were collected on day zero (D0) and after 7 days of therapy (D7). Evaluation of the cellular receptors was carried out by flow cytometry. Expression of CD14 on monocytes and of CD11b and CXCR2 on neutrophils from SP was lower than that from HV. Conversely, expression of TLR5 on monocytes and neutrophils was higher in SP compared with HV. Expression of TLR2 on the surface of neutrophils and that of TLR5 on monocytes and neutrophils of SP was lower at D7 than at D0. In addition, SP who survived showed reduced expression of TLR2 and TLR4 on the surface of neutrophils at D7 compared to D0. Expression of CXCR2 for surviving patients was higher at follow-up compared to baseline. We conclude that expression of recognition and cell signaling receptors is differentially regulated between SP and HV depending on the receptor being evaluated.
\end{abstract}

Key words: Sepsis; Toll-like receptors; TLR5; TLR9; CXCR2

\section{Introduction}

Sepsis has been defined as a systemic inflammatory response syndrome triggered by infection (1). However, a state of sepsis-induced immunosuppression is increasingly demonstrated in experimental $(2,3)$ and clinical sepsis (4). It has now been recognized that most patients dying from sepsis present evidence of unresolved septic foci and immunosuppression (5). We found that decreased or exacerbated responses are observed over the course of the clinical manifestations, depending on the functions and cells evaluated (6).
Bacterial sensing and cell signaling involve complex mechanisms and are modulated during sepsis (7). Specialized phagocytes, such as neutrophils and monocytes, play an important role in host defense and are critical cellular components of the innate immune system. These cells have the ability to recognize and engulf pathogens, responding with the production of inflammatory mediators and coordinating additional mechanisms, such as the recruitment of polymorphonuclear cells from the peripheral blood to the site of infection, ultimately 
leading to the resolution of the infection. The immune response to bacterial infection is triggered when pattern recognition receptors on phagocytes, including the Tolllike receptors (TLRs), recognize the pathogen-associated molecular patterns (PAMPs) expressed by the infecting microorganism (8).

TLRs are expressed both on the cell surface and intracellularly and recognize PAMPs as well as endogenous mediators (8). TLR1, TLR2, TLR4, TLR5, and TLR6 are expressed on the cell surface, whereas TLR3, TLR7, TLR8, and TLR9 are located in intracellular compartments (9).

There is some specificity between TLRs and the microbial structures that they recognize. TLR2 is a promiscuous receptor that recognizes multiple ligands expressed on a variety of microorganisms, acts in conjunction with TLR1 or TLR6, and is stimulated by bacterial cell wall peptidoglycan (PGN) and lipoteichoic acid (9). TLR4/myeloid differentiation protein-2, in association with the coreceptor CD14, recognizes lipopolysaccharide (LPS) (7). CD14 is present in two forms: membrane-bound CD14 (mCD14) and soluble CD14 (sCD14). CD14 plays a major role in LPS recognition, and it was recently shown to also recognize Gram-positive bacteria (10). TLR5 is activated by bacterial flagellin and TLR9, which is intracellular, is involved in the recognition sequence of bacterial DNA (CpG oligodeoxynucleotides) (9). All of these TLRs signal via the adaptor molecule myeloid differentiation primary response 88 (MyD88) gene, although TLR4 may alternatively signal through an MyD88-independent pathway $(7,9)$. TLRs function as dimers and often use coreceptors, such as CD14, to assist in pathogen recognition. Specifically, CD14 interacts with TLR4- and TLR2-containing dimeric complexes to transduce activation signals in response to bacterial pathogens (11).

Recognition of pathogen components and the subsequent activation of intracellular signaling pathways lead to the synthesis and release of proinflammatory cytokines, such as tumor necrosis factor-alpha (TNF- $\alpha$ ), interleukin (IL)-1, and IL-8, as well as anti-inflammatory cytokines, such as IL-10 (7). The expression patterns of TLRs in different cell types may be an important regulatory mechanism of the innate immune response to different pathogens.

The mechanism by which leukocytes clear microorganisms is a complex process that involves the production of chemokines, rolling, adhesion, and cell migration to the site of infection (12). This process involves chemokine receptors, such as CXCR2 (13), and the expression of receptors, such as CD11b and CD66b, which are involved in the activation, migration, and adhesion of neutrophils (14).

This study tested the hypothesis that the regulation of the cellular functions of monocytes and neutrophils that occurs during sepsis may partly reflect changes in the expression of cellular receptors that are involved in the recognition of microorganisms, cell signaling, chemotaxis, and cellular adhesion. Thus, we evaluated the dynamics of CD14 expression on the surface of monocytes; CD66b and CXCR2 on the surface of neutrophils; and CD11b, TLR2, TLR4, TLR5, and TLR9 in both cell populations during the progression of sepsis.

\section{Material and Methods}

\section{Patients and healthy volunteers}

Patients older than 18 years were included in the first $72 \mathrm{~h}$ of diagnosis of sepsis or $48 \mathrm{~h}$ following the first organ dysfunction (severe sepsis) or refractory hypotension (septic shock). Patients were excluded from the study if they were infected with human immunodeficiency virus, had any neoplastic or immunosuppressive disease, or were participating in any other study protocol. Patients were enrolled in the intensive care units of three general hospitals (São Paulo, Sírio Libanês, and Israelita Albert Einstein) located in São Paulo, Brazil, between January 2008 and December 2009. One healthy control was included for every two patients. At the three different sites, patients were monitored for age and gender. They were grouped according to age and gender, and, for each pair of patients, one healthy volunteer of the same gender and with age in the range of 5 years from the patient's age was enrolled. In cases of patients older than 65 years, a control older than 65 years was accepted. The study was approved by the Institutional Review Boards of these three institutions. Written informed consent was obtained from all participants or, in cases in which that was not possible, from relatives before enrollment in the study protocol.

\section{Blood sampling}

Samples were obtained from septic patients at admission [day zero (D0), $n=77$ )] and after 7 days of therapy $(D 7, n=45)$. Forty healthy volunteers were enrolled as the control group. Five milliliters of blood was drawn from both the healthy volunteers and the septic patients into EDTA-treated tubes (Becton Dickinson, UK). Samples were processed within $4 \mathrm{~h}$ at the Laboratório de Imunologia, Divisão de Doenças Infecciosas, Escola Paulista de Medicina, Universidade Federal de São Paulo, Brazil.

\section{Immunophenotyping of monocytes and neutrophils}

The expression of cell surface and intracellular receptors was investigated in whole blood samples. A total of $100 \mu \mathrm{L}$ whole blood from patients and healthy volunteers was transferred to polystyrene tubes (Becton Dickinson, USA) and stained with the following monoclonal antibodies or isotype controls: $5 \mu \mathrm{L}$ CD66bfluorescein isothiocyanate (FITC), $10 \mu \mathrm{L}$ mlgG $_{2}$-phycoerythrin (PE), $5 \mu \mathrm{L}$ CD14-peridinin chlorophyll protein (PerCP), and $2 \mu \mathrm{L} \mathrm{mlgG}_{2 \mathrm{~b}}$-allophycocyanin (APC) (Tube 1); $5 \mu \mathrm{L}$ CD66b-FITC, $10 \mu \mathrm{L}$ TLR2-PE, $5 \mu \mathrm{L}$ CD14-PerCP, and 
$3 \mu \mathrm{L}$ CXCR2-APC (Tube 2); $5 \mu \mathrm{L}$ CD66b-FITC, $20 \mu \mathrm{L}$ TLR4-PE, $5 \mu \mathrm{L}$ CD14-PerCP, and $2 \mu \mathrm{L}$ CD11b-APC (Tube 3); $5 \mu \mathrm{L}$ CD14-PerCP and $3 \mu \mathrm{L}$ CD15-APC (Tube 4); and $6 \mu \mathrm{L}$ TLR5-FITC, $5 \mu \mathrm{L}$ CD14-PerCP, and $3 \mu \mathrm{L}$ CD15-APC (Tube 5) (Becton Dickinson). Samples were incubated with fluorochrome-conjugated monoclonal antibodies for surface staining for $15 \mathrm{~min}$ in the dark at room temperature. Two milliliters of lysis solution (Becton Dickinson) was added into each tube and the samples were incubated for $10 \mathrm{~min}$ in the dark at room temperature, centrifuged at $3184 \mathrm{~g}$ for $5 \mathrm{~min}$ at $4^{\circ} \mathrm{C}$, and washed with $2 \mathrm{~mL}$ phosphate-buffered saline (PBS; $0.15 \mathrm{M}$ PBS: $8.0 \mathrm{~g} \mathrm{NaCl}, 0.2 \mathrm{~g} \mathrm{KH}_{2} \mathrm{PO}_{4}, 1.15 \mathrm{~g}$ $\mathrm{Na}_{2} \mathrm{HPO}_{4}$, and $0.2 \mathrm{~g} \mathrm{KCl}$, in distilled water to $1 \mathrm{~L}, \mathrm{pH} 7.2$ ). Tubes 1, 2, and 3 were suspended in $0.3 \mathrm{~mL}$ PBS with $1 \%$ sodium azide (Sigma, USA) and stored at $2-8^{\circ} \mathrm{C}$ until use. Tubes 4 and 5 were suspended with $50 \mu \mathrm{L}$ permeabilization buffer (PBS with $5 \%$ saponin; Sigma). Tube 5 received $1.5 \mu \mathrm{L}$ TLR9-PE and was incubated at $2-8^{\circ} \mathrm{C}$ in the dark for $30 \mathrm{~min}$. The samples were washed with $2 \mathrm{~mL}$ PBS, and the cells were suspended in $0.3 \mathrm{~mL}$ PBS with $1 \%$ sodium azide and analyzed by flow cytometry.

\section{Acquisition and analysis of flow cytometry data}

Flow cytometry was performed with a FACSCalibur four-color flow cytometer (Becton Dickinson). A total of 5000 events were acquired for monocytes, combining side scatter and positivity for CD14 staining. To analyze cell surface and intracellular receptors, monocytes were defined by combining forward scatter and side scatter parameters and positivity for CD14, while neutrophils were defined by combining forward scatter and side scatter parameters and positivity for CD66b (15) or positivity for CD15. Data analysis of the expression of TLR2, TLR4, TLR5, TLR9, CD11b, CXCR2, CD66b, and CD14 receptors was carried out using the FlowJo software (Tree Star, USA).

Expression of TLR2, TLR4, TLR5, TLR9, and CD11b was evaluated on monocytes and neutrophils, expression of CD14 was assessed on monocytes, and expression of CD66b and CXCR2 was analyzed on neutrophils. Receptor expression was measured as the geometric mean fluorescence intensity (GMFI), and the results are reported as the difference between the fluorescence obtained with the specific antibodies and isotype controls.

\section{Statistical analysis}

The Kolmogorov-Smirnov test was applied to verify whether a distribution was normal. Continuous variables are reported as means $\pm S D$ when the distribution was normal or as the median and range when the distribution was not normal. Discrete variables are reported as percentages. Differences among the groups were tested with the Mann-Whitney U-test, and comparisons between patient samples (D7 vs D0) were performed using the Wilcoxon signed-rank test. $\mathrm{P} \leq 0.05$ was considered to be significant. These analyses were performed using SPSS package 13.0 (SPSS Inc. and Predictive Analytics, USA).
Table 1. Demographic data and outcomes from septic patients included in the study.

\begin{tabular}{|c|c|}
\hline \multicolumn{2}{|c|}{ Cohort of septic patients $(n=77)$} \\
\hline Age (years) & $61 \pm 17.2$ \\
\hline \multicolumn{2}{|l|}{ Gender } \\
\hline Male & $43(55.8 \%)$ \\
\hline Female & $34(44.2 \%)$ \\
\hline \multicolumn{2}{|l|}{ Stages of sepsis } \\
\hline Sepsis & $4(5.2 \%)$ \\
\hline Severe sepsis & $18(23.4 \%)$ \\
\hline Septic shock & $55(71.4 \%)$ \\
\hline SOFA score & $7.8 \pm 4$ \\
\hline APACHE II score & $18 \pm 6.1$ \\
\hline \multicolumn{2}{|l|}{ Inhospital mortality } \\
\hline Survivors & $50(64.9 \%)$ \\
\hline Nonsurvivors & $27(35.1 \%)$ \\
\hline \multicolumn{2}{|c|}{ Outcome according to stage at enrollment } \\
\hline \multicolumn{2}{|c|}{ Sepsis } \\
\hline Survivors & $4(100 \%)$ \\
\hline Nonsurvivors & $0(0 \%)$ \\
\hline \multicolumn{2}{|l|}{ Severe sepsis } \\
\hline Survivors & $11(61.1 \%)$ \\
\hline Nonsurvivors & $7(38.9 \%)$ \\
\hline \multicolumn{2}{|l|}{ Septic shock } \\
\hline Survivors & $35(63.6 \%)$ \\
\hline Nonsurvivors & $20(36.4 \%)$ \\
\hline \multicolumn{2}{|l|}{ Sources of infection } \\
\hline Respiratory tract & $29(37.7 \%)$ \\
\hline Abdomen & $20(26.0 \%)$ \\
\hline Urinary tract & $13(16.9 \%)$ \\
\hline Skin or soft tissue & $5(6.4 \%)$ \\
\hline Others & $10(13.0 \%)$ \\
\hline
\end{tabular}

Data are reported as means \pm SD or number with percent in parentheses. SOFA: sepsis-related organ failure assessment; APACHE II: acute physiology and chronic health evaluation.

\section{Results}

\section{Demographic data}

The study included a cohort of 77 septic patients who were admitted to the intensive care units at three large tertiary hospitals located in the city of São Paulo, Brazil, and who met the criteria for sepsis $(n=4)$, severe sepsis $(n=18)$, or septic shock $(n=55)$, as previously described by Bone et al. (1). Demographic data from patients are shown in Table 1. Forty healthy volunteers matched for age and gender to the septic patients were included in the study. The mean age of the healthy volunteers was $56.9 \pm 16$ years and $23(57.5 \%)$ were males.

The etiology of sepsis was considered as the microorganisms recovered from the primary site of infection or in blood cultures. The agents causing sepsis were identified in almost $50 \%$ of the cases: $15.6 \%$ of sepsis were caused by Gram-positive bacteria, $29.9 \%$ by 

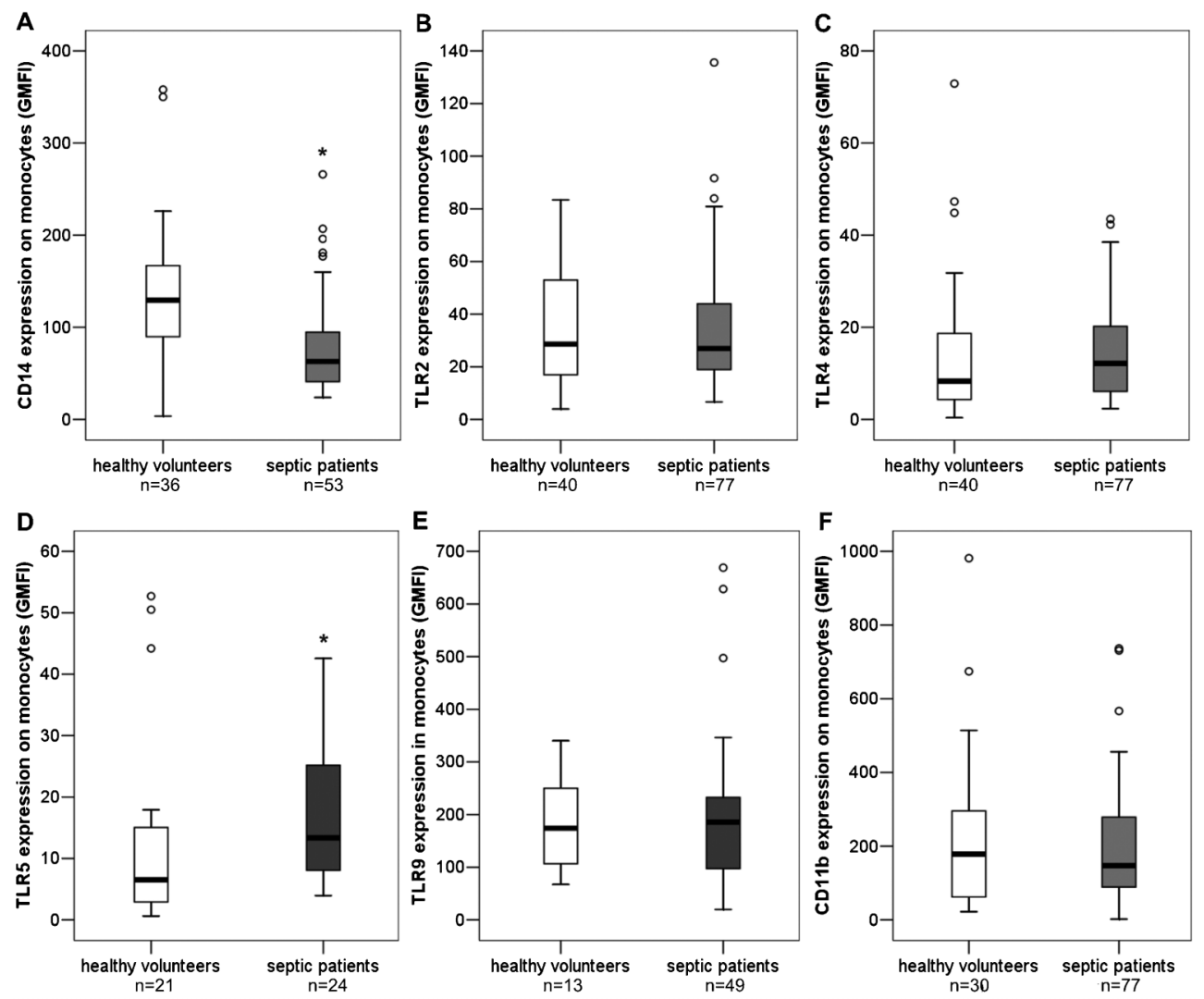

Figure 1. Analysis of the expression of surface and intracellular receptors on monocytes from healthy volunteers and septic patients. Receptor expression was analyzed in histograms and reported as the geometric mean fluorescence intensity (GMFI): CD14 (A), TLR2 $(B)$, TLR4 $(C)$, TLR5 $(D)$, TLR9 $(E)$, and CD11b $(F)$. Data are reported as box plots, medians with 25 to $75 \%$ quartiles (box) and minimum and maximum values (bars). Empty circles indicate outliers. ${ }^{*} \mathrm{P}<0.05$ compared to healthy volunteers (Mann-Whitney U-test).

Gram-negative bacteria, and $2.6 \%$ were mixed infections; in the remaining cases $(51.9 \%)$, the etiology could not be determined.

\section{Expression of receptors in septic patients and healthy volunteers}

Recognition and signaling receptors. The expression of CD14 on the surface of monocytes was significantly lower in the septic patients compared to the healthy volunteers $(P=0.001$; Figure $1 A)$.

There was no difference in the expression of TLR2 on the surface of monocytes $(P=0.915$; Figure $1 B)$ or neutrophils $(P=0.615$; Figure $2 A)$ in the septic patients compared to the healthy volunteers. No difference in the expression of TLR4 on the surface of monocytes was observed between septic patients and healthy volunteers $(P=0.084$; Figure $1 C)$. The expression of this receptor on neutrophils was similar for septic patients and healthy volunteers as well $(\mathrm{P}=0.556$; Figure $2 \mathrm{~B})$. The expression of TLR5 on the surface of monocytes in the septic patients was significantly higher compared to that in the healthy volunteers ( $P=0.041$; Figure 1D). Similarly, TLR5 expression on neutrophils in the septic patients was higher than on neutrophils in the healthy volunteers $(P=0.001$; Figure $2 \mathrm{C})$. There was no difference in the expression of intracellular TLR9 in the monocytes of septic patients and healthy volunteers $(P=0.762$; Figure $1 E)$. The same results were observed for the neutrophils of septic patients and healthy volunteers $(P=0.355$; Figure 2D).

Chemotaxis and adhesion receptors. There was no difference in the expression of CD11b on the surface of monocytes between septic patients and healthy volunteers $(P=0.771$; Figure $1 F)$, although $C D 11 b$ expression was lower on the surface of neutrophils from septic patients compared to healthy volunteers $(P=0.007$; Figure 2E). Expression of CXCR2 was also lower on neutrophils from the former group $(P=0.001$; Figure $2 F)$. No difference in the expression of CD66b on the surface of neutrophils was found between the septic patients and healthy volunteers (median $\mathrm{GMFI}=102.00$, range $=33.00$ 476.00, and median $\mathrm{GMFI}=95.25$, range $=10.90-2457.00$, respectively, $\mathrm{P}=0.769$ ). 

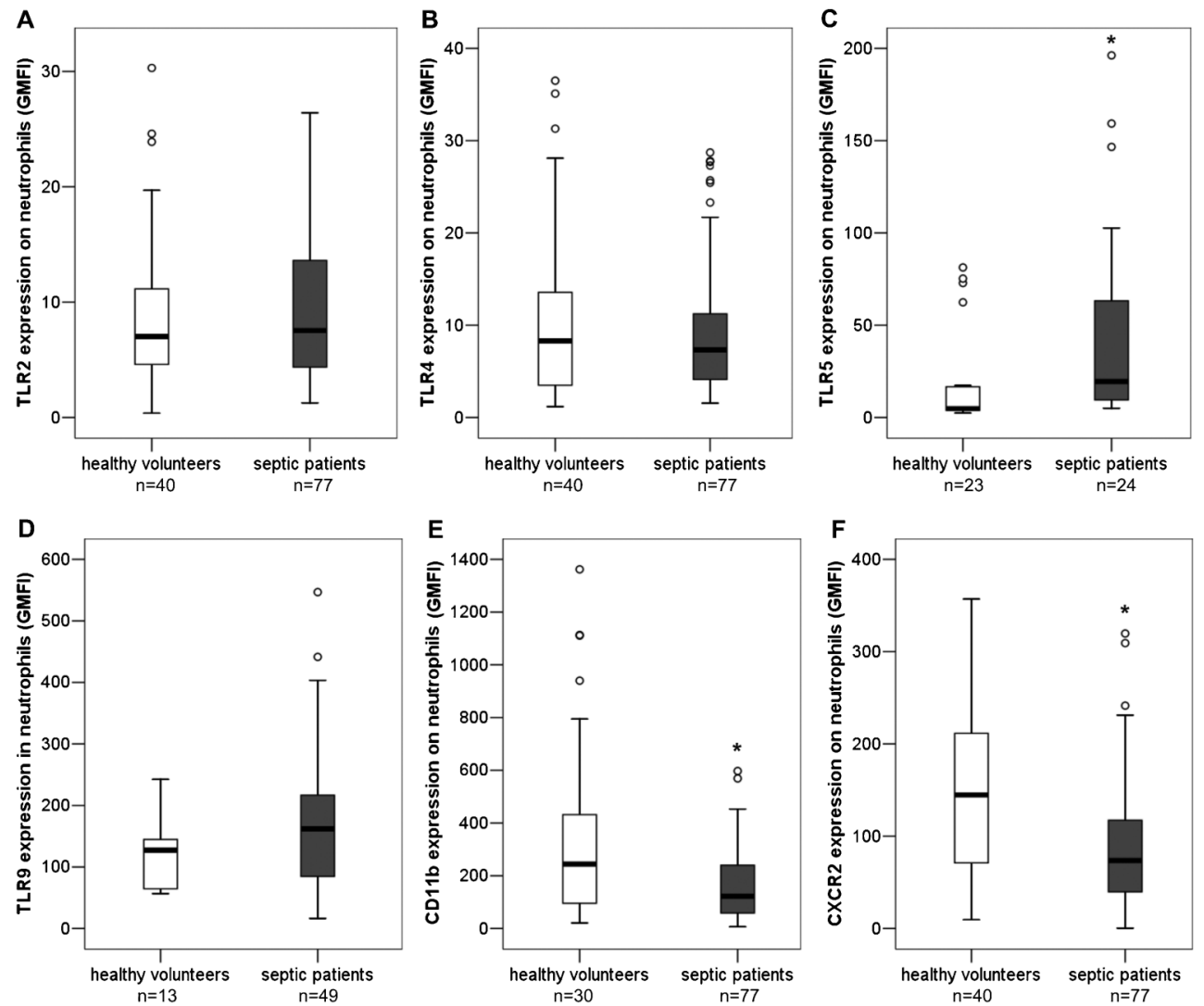

Figure 2. Analysis of the expression of surface and intracellular receptors on neutrophils from healthy volunteers and septic patients. Receptor expression was analyzed in histograms and reported as the geometric mean fluorescence intensity (GMFI): TLR2 ( $A$ ), TLR4 $(B)$, TLR5 $(C)$, TLR9 $(D)$, CD11b $(E)$, and CXCR2 $(F)$. Data are reported as box plots, medians with 25 to $75 \%$ quartiles (box) and minimum and maximum values (bars). Empty circles indicate outliers. ${ }^{*} \mathrm{P}<0.05$ compared to healthy volunteers (Mann-Whitney U-test).

\section{Expression of receptors at follow-up for septic patients}

Recognition and signaling receptors. There was no difference in the expression of CD14 on the surface of the monocytes of septic patients at admission (D0) compared to samples collected at D7 (Table 2). The expression of TLR2 on the surface of monocytes did not differ between the D0 and D7 samples; however, its expression on the surface of neutrophils was decreased at D7 compared to D0 (Table 2). TLR4 expression on the surface of monocytes and neutrophils did not differ between the D0 and D7 samples (Table 2). Additionally, intracellular expression of TLR9 in both cell types did not change in the septic patients at follow-up. However, the expression of TLR5 on the surface of monocytes and neutrophils decreased after the 7 days of therapy (Table 2).

Chemotaxis and adhesion receptors. There was no difference in the expression of CD66b on the surface of neutrophils at enrollment or after 7 days at follow-up (Table 2). Additionally, no difference in the expression of CD11b on the surface of monocytes and neutrophils of septic patients was observed between D0 and D7 (Table 2).
Furthermore, the expression of CXCR2 on the surface of neutrophils did not differ at follow-up (Table 2).

\section{Expression of receptors in septic patients according to outcome}

Recognition and signaling receptors. Expression of recognition and signaling receptors was evaluated in the samples from septic patients taken at follow-up and stratified according to outcome.

There was no difference in the expression of CD14 on the surface of monocytes from surviving patients at enrollment (D0; median $\mathrm{GMFI}=62.00$, range $=29.60$ 181.00) and follow-up (D7; median GMFI $=64.00$, range $=33.90-155.0 ; P=0.903$ ). Additionally, no differences were observed for the nonsurvivors between samples at admission (D0; median $\mathrm{GMFI}=68.15$, range $=54.00-241.00$ ) and follow-up (median $\mathrm{GMFI}=$ 73.65, range $=30.60-183.00 ; P=0.646)$.

Expression of TLR2 on the surface of the neutrophils of surviving patients was significantly lower at D7 compared to that at $D 0(P=0.001$; Figure $3 A)$, while TLR2 expression on monocytes did not change in these 
Table 2. Expression of receptors at baseline and follow-up of the septic patients.

\begin{tabular}{lccc}
\hline & $\mathrm{n}$ & $\mathrm{D} 0$ & $\mathrm{D} 7$ \\
\hline Monocytes & & & $67.35(30.60-183.00)$ \\
CD14 & 32 & $64.25(29.6-266.00)$ & $23.02(6.37-107.20)$ \\
TLR2 & 45 & $25.65(6.64-135.60)$ & $11.36(1.82-83.70)$ \\
TLR4 & 45 & $12.19(2.35-43.51)$ & $8.97(2.49-47.51)^{\star}$ \\
TLR5 & 12 & $15.15(3.96-42.55)$ & $160.43(48.82-398.90)$ \\
TLR9 & 25 & $175.13(19.71-668.92)$ & $188.5(21.37-947.19)$ \\
CD11b & 45 & $137.92(1.74-736.70)$ & $5.09(0.62-28.01)^{\star}$ \\
Neutrophils & & & $5.37(0.97-30.37)$ \\
TLR2 & 45 & $7.53(1.27-26.42)$ & $14.87(2.89-87.69)^{\star}$ \\
TLR4 & 45 & $30.62(1.55-28.72)$ & $142.40(34.75-299.04)$ \\
TLR5 & 12 & $150.49(16.48-441.47)$ & $78.0(19.60-261.00)$ \\
TLR9 & 25 & $86.65(33.00-476.00)$ & $105.44(12.33-965.69)$ \\
CD66b & 32 & $122.96(6.36-597.21)$ & $84.51(6.45-317.39)$ \\
CD11b & 45 & $66.68(0.37-319.63)$ & \\
CXCR2 & 45 & &
\end{tabular}

Data are reported as median percent and range of geometric mean fluorescence intensity. D: day. ${ }^{*} \mathrm{P}<0.05$ compared to D0 (Wilcoxon signed-rank test).

patients (median $\mathrm{GMFI}=26.86$, range $=6.64-135.60$; median $\mathrm{GMFI}=23.90$, range $=6.37-72.34$; $\mathrm{D} 0$ and $\mathrm{D} 7$, respectively; $P=0.127)$. Expression of TLR2 on the surface of monocytes (median $\mathrm{GMFI}=25.32$, range $=7.82-83.98$; median $\mathrm{GMFI}=19.37$, range $=9.21-107.20$; $\mathrm{D} 0$ and $\mathrm{D} 7$, respectively; $P=0.609$ ) and neutrophils did not change at follow-up in nonsurvivors $(P=0.796$; Figure $3 A)$. Expression of TLR4 on the surface of neutrophils from patients that survived decreased significantly at D7 compared to D0 $(P=0.020)$, while no difference was found in nonsurvivors $(P=0.501$; Figure 3B). Additionally, no difference was found in the expression of TLR4 on monocytes between D0 and
D7 from surviving septic patients (median $\mathrm{GMFI}=12.60$ range $=2.35-38.50 ;$ median $\mathrm{GMFI}=10.34$, range $=1.82$ 83.70; $D 0$ and D7, respectively; $P=0.922$ ) or nonsurvivors (median $\mathrm{GMFI}=10.66$, range $=2.85-43.51$; median $\mathrm{GMFI}=13.66$, range $=2.51-31.00 ; \mathrm{D} 0$ and $\mathrm{D} 7$, respectively; $\mathrm{P}=0.756$ ). There was no difference in the expression of intracellular TLR9 between D0 and D7 in survivors for both monocytes (median GMFI $=153.43$, range $=19.71-668.26$; median $\mathrm{GMFI}=160.43$, range $=58.10-398.90$; D0 and D7, respectively) and neutrophils (median $\mathrm{GMFI}=31.37$, range $=16.48-441.47 ;$ median $\mathrm{GMFI}=142.76$, range $=34.75-250.78 ; \mathrm{D} 0$ and $D 7$, respectively; $P=0.233$
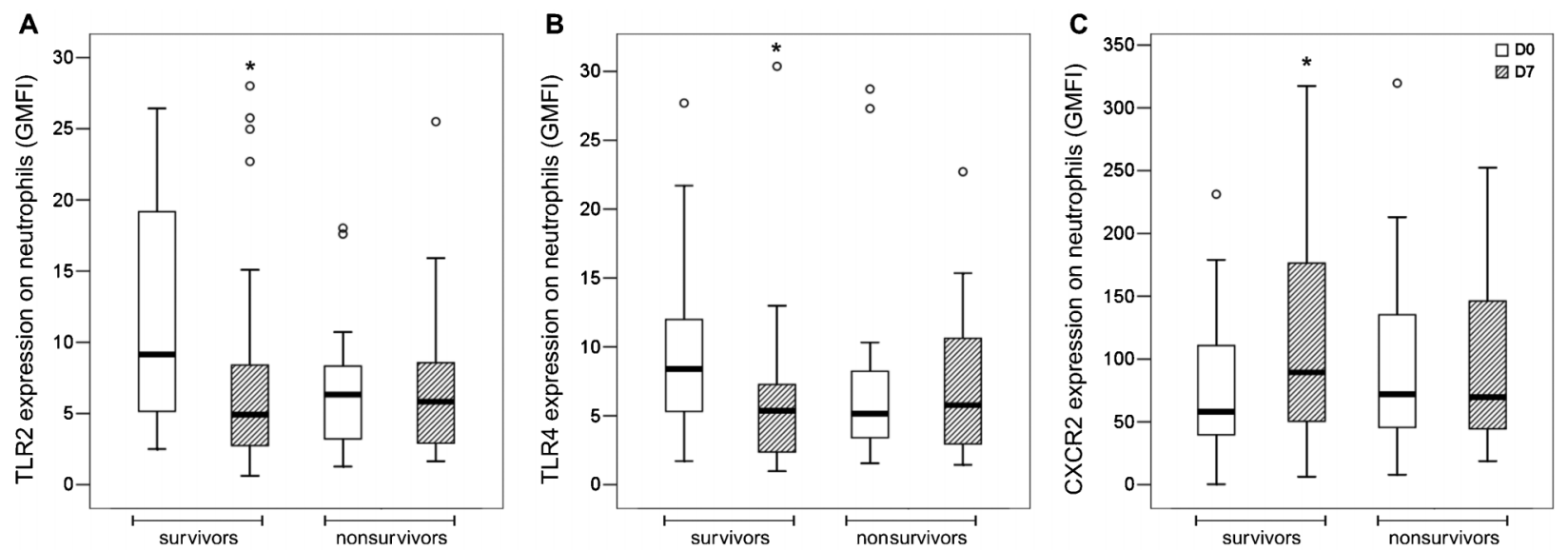

Figure 3. Expression of TLR2, TLR4, and CXCR2 surface receptors on neutrophils from surviving septic patients $(n=29)$ and nonsurvivors $(n=16)$ at $D 0$ and D7. Surface marker expression was analyzed in histograms and reported as the geometric mean fluorescence intensity (GMFI): TLR2 $(A)$, TLR4 $(B)$ and CXCR2 $(C)$. Data are reported as box plots, medians with 25 to $75 \%$ quartiles (box) and minimum and maximum values (bars). Empty circles indicate outliers. D: day. ${ }^{*} \mathrm{P}<0.05$ compared to D0 from the same group (Wilcoxon signed-rank test). 
and $\mathrm{P}=0.955)$. Furthermore, there was no difference in the expression of TLR9 in monocytes and neutrophils in the nonsurvivor group at D0 (median GMFI=206.68, range $=97.21-497.33$; median $\mathrm{GMFI}=186.96$, range $=$ 84.57-546.72, respectively) and D7 (median GMFI= 165.12, range $=48.82-280.48$; median $\mathrm{GMFI}=136.34$, range $=45.90-299.00$, respectively; $\mathrm{P}=0.059$ and $\mathrm{P}=0.203$ for monocytes and neutrophils, respectively).

Chemotaxis and adhesion receptors. The expression of CD66b on the surface of neutrophils from survivors (median $\mathrm{GMFI}=84.70$, range $=45.90-476.00$; median $\mathrm{GMFI}=78.90$, range $=19.60-261.00 ; \mathrm{D} 0$ and $\mathrm{D} 7$, respectively) and nonsurvivors (median $\mathrm{GMFI}=136.50$, range $=33.00-241.00 ;$ median $\mathrm{GMFI}=57.95$, range $=48.90-136.00$; $D 0$ and $D 7$, respectively) did not change between D0 and D7 $(P=0.498$ and $P=0.139$, respectively, for survivors and nonsurvivors). Expression of CD11b on the surface of monocytes did not change between D0 (median $\mathrm{GMFI}=137.86$, range $=1.74$ 393.75) and D7 (median GMFI=188.19, range $=23.43$ 549.40) in survivors $(P=0.294)$. Similar results were observed for expression of this receptor on the surface of neutrophils from survivors (median $\mathrm{GMFI}=110.74$, range $=6.36-423.52$; median $\mathrm{GMFI}=92.32$, range $=$ 23.87-423.52; D0 and D7, respectively; $P=0.304$ ). Additionally, expression of this receptor did not change at follow-up for nonsurvivors when both cell populations, monocytes (median $\mathrm{GMFI}=159.24$, range $=35.34$ 736.70; median $\mathrm{GMFI}=302.57$, range $=21.37-947.19$; D0 and D7, respectively; $\mathrm{P}=0.301$ ), and neutrophils (median $\mathrm{GMFI}=135.60$, range $=35.94-453.00$; median $\mathrm{GMFI}=170.84$, range $=12.33-965.69 ; \mathrm{D} 0$ and $\mathrm{D} 7$, respectively; $P=0.569$ ) were evaluated. Expression of CXCR2 on the surface of neutrophils was higher at D7 compared to $D 0$ for the patients that survived $(P=0.031)$. However, the expression of this receptor on the surface of neutrophils did not change at follow-up for nonsurvivors $(P=0.952$; Figure 3C).

\section{Discussion}

Our results demonstrate that the cellular receptors expressed on neutrophils and monocytes that are involved in pathogen recognition, cell signaling, migration, and adhesion to endothelial cells are differentially regulated in septic patients compared to healthy volunteers.

In the present study, the expression of mCD14 on the surface of monocytes was lower in septic patients compared to healthy volunteers, which corroborates previous reports $(16,17)$. We and others found decreased expression of mCD14 on monocytes and higher levels of sCD14 in plasma from septic patients compared to healthy volunteers (16). Conversely, preserved mCD14 expression on monocytes and even increased expression on neutrophils from septic patients have been reported $(18,19)$.
Modulation of the expression of mCD14 on monocytes from septic patients is consistent with its modulation in in vitro experiments. Jorgensen et al. (20) reported that PGN increased the expression of CD14, while LPS decreased its expression. In a previous study, we found a biphasic pattern of CD14 modulation following LPS stimulation, with increased expression in the first $6 \mathrm{~h}$ of stimulation followed by decreased expression after 6 and $24 \mathrm{~h}$ of incubation (21). The changes in CD14 expression that are observed with sepsis and in LPS in vitro experiments illustrate that the cellular response to LPS and other products that use CD14 as a pattern recognition receptor may be modulated during infection.

CD66b is a glycoprotein involved in the activation, adhesion, and migration of neutrophils (14). In the present study, the expression of CD66b did not differ between all conditions studied. This result differs from a previously published study from our group (15), which showed higher expression of CD66b on the surface of neutrophils of septic patients compared to healthy volunteers and the results obtained by Muller Kobold et al. (22), which demonstrated that CD66b is differentially expressed in septic patients according to outcome. This discrepancy may reflect differences in the populations studied and the complexity of immune regulation in sepsis.

During the last few years, many studies evaluating TLR pathways in the context of sepsis have been published (reviewed in Refs. 6 and 7). In the present study, expression of TLR2 and TLR4 on the surface of neutrophils and monocytes did not differ between septic patients and healthy volunteers. The lack of modulation of TLR2 and TLR4 expression on monocytes and neutrophils is in agreement with previous studies from our group that included patients in different stages of sepsis $(15,16)$. In contrast to our findings, others have found an upregulation of TLR2 expression on monocytes and neutrophils in septic patients compared to the control group (23). Despite the upregulation of TLR2, as well as TLR4, no differences were found in response to TLR2 and TLR4 agonists in septic patients in that study (23). Expression of TLR2 on the surface of neutrophils of septic patients decreased after 7 days at follow-up in our study. Interestingly, expression of this receptor on neutrophils decreased in the patients who survived while expression remained stable in those who died. Additionally, expression of TLR4 on neutrophils decreased at follow-up in survivors, while no differences were observed in the whole cohort. Decreased expression of TLR2 and TLR4 on the surface of neutrophils of survivors might be associated with the control of inflammation. In contrast, Schaaf et al. (24) showed an association between death and decreased expression of TLR2 on the surface of monocytes and lower production of IL-6, TNF- $\alpha$, and IL-10 in septic patients. Thus, it is difficult to conclude whether the dynamics of TLR2 expression on monocytes and neutrophils from septic patients influences the prognosis 
of these patients and which mechanisms are involved.

TLR5 recognizes bacterial flagellin and is expressed in different cell types, including monocytes, mastocytes, dendritic cells, and epithelial cells $(8,9)$. It is interesting that flagellin does not contain an obvious feature that defines it as non-self or as a pathogen-associated structure (8). Some studies demonstrate that TLR5 plays a crucial role in protecting the intestine from pathogens. For example, TLR5-depleted mice develop exacerbated colitis in a model of spontaneous colitis (25). In the present study, the expression of TLR5 was higher among septic patients compared to healthy volunteers; in addition, TLR5 expression decreased after 7 days at follow-up for septic patients.

TLR9 recognizes unmethylated CpG motifs that are present in bacterial DNA (26). Similar to TLR3 and TLR7, TLR9 recognizes microbial and modified nucleic acids in the endosome (9). Expression of TLR9 did not differ among septic patients and healthy volunteers in our study, and the sample size was small for evaluating expression of TLR9 according to outcome. Experimental studies support an important role for TLR9 in sepsis. Plitas et al. (27) demonstrated increased bacterial clearance in TLR9 $^{-1}$ mice subjected to cecal ligation and puncture CLP-related peritonitis compared with wild-type animals. The authors also showed increased survival in wild-type animals when an inhibitory $\mathrm{CpG}$ sequence that blocks TLR9 was administered just before CLP. Another study employing the CLP model showed that TLR9 ${ }^{-1-}$ mice do not demonstrate neutrophil migration failure and present a low systemic inflammatory response and a high survival rate (28).

Thus, regarding TLR expression, TLR5 was increased on monocytes and neutrophils from septic patients and TLR2 and TLR4 were decreased on neutrophils at followup for surviving patients. However, despite these changes, it is likely that the functional changes in monocytes and neutrophils that are observed during sepsis are not directly linked to the modulation of expression of TLR (reviewed in Refs. 6 and 7). This is consistent with previous results obtained by our group and others. We found a dynamic modulation of cytokine production by monocytes (16) and reactive oxygen species generation by monocytes and neutrophils $(29,30)$ during the different stages of sepsis that is not associated with the expression of TLR2 and TLR4. The lower production of inflammatory cytokines by monocytes from septic patients resembles the functional changes seen in alternatively activated macrophages; accordingly, increased expression of markers of alternative activation - CD166 and CD206 - were found on monocytes from septic patients (31). Furthermore, we found that TLR signaling pathway genes are regulated differently in mononuclear cells and neutrophils of septic patients. Mononuclear cells presented downregulation in septic shock, predominantly in the nuclear factor kappa $\mathrm{B}(\mathrm{NF} \kappa \mathrm{B})$ pathway, while neutrophils showed predominantly upregulated genes throughout the stages of sepsis (32). These and other studies support the intracellular regulation of TLR cell signaling in sepsis (6), which is corroborated by studies of LPS-induced tolerance. Accordingly, the modulation of gene expression in tolerant cells in response to LPS appears to occur independently of changes in TLR expression, as demonstrated by Foster et al. (33) and by a study from our group (34). Integrins are crucial to leukocyte migration during the inflammatory response. The expression of CD11b on the surface of neutrophils was lower among the septic patients compared to the healthy volunteers in our cohort, which is in agreement with previous results (19). The lower expression of CD11b on neutrophils could be a result of receptor internalization, as reported by previous studies (35). Tansho-Nagakawa et al. (19) suggested that the decreased CD11b expression observed in septic patients might also be due to an increased percentage of circulating immature neutrophils. In contrast, Lin et al. (36) found increased expression of CD11b on the surface of neutrophils of septic patients compared to healthy volunteers. We did not find differences in CD11b expression between survivors and nonsurvivors in this study, which contrasts with a study by Muller Kobold et al. (22), which showed lower expression of CD11b on the neutrophils of septic patients who died compared with those who survived. The expression of CD11b on monocytes did not differ between the septic patients and healthy volunteers, which is in agreement with our previous study on severe sepsis and septic shock patients (16). In contrast, LPS induces the expression of CD11b on human monocytes and neutrophils in vitro (21) and on monocytes following intravenous injection in healthy volunteers (37).

CXCR2 is a chemokine receptor involved in neutrophil migration to sites of injury. In the present study, we observed lower CXCR2 expression in the neutrophils of septic patients compared to healthy volunteers. These results are in agreement with previous results (38). The decreased expression of CXCR2 may occur in response to the high levels of circulating chemokines in a possible negative-feedback mechanism that could be present in patients with advanced stages of sepsis (38). Additionally, a previous study published by our group demonstrated lower expression of this receptor on the surface of neutrophils from healthy volunteers after stimulation with LPS in vitro (21), and Juffermans et al. (39) reported that the administration of low doses of LPS in vivo induces a decrease in both the CXCR1 and CXCR2 receptors on circulating granulocytes in the whole blood of healthy individuals. Reduced neutrophil migration to the site of infection is associated with a worse prognosis during sepsis. This reduced expression may be an attempt by the host to limit excessive inflammation induced by granulocytes at the site of infection but may also be detrimental. A study from Rios-Santos et al. (40) demonstrated that mice subjected to CLP show deficient neutrophil migration to 
the site of infection during severe sepsis, which is associated with decreased expression of CXCR2 on the cell surface. In the present study, the expression of CXCR2 differed between survivors and nonsurvivors at follow-up. CXCR2 expression was higher on the surface of neutrophils on D7 for survivors, suggesting that reversal of the downregulation of expression of this receptor may be important to restore neutrophil function.

In conclusion, a dynamic modulation of cell surface receptor expression was found when septic patients were compared to healthy volunteers. The expression of CXCR2 and CD11b was lower, while the expression of TLR5 was higher on the surface of neutrophils in septic patients compared to healthy volunteers. With regard to monocytes, CD14 expression was lower and TLR5 expression was higher in septic patients compared with healthy volunteers. When evaluated at follow-up, expression of TLR2 and TLR5 on neutrophils and expression of TLR5 on monocytes were

\section{References}

1. Bone RC, Sibbald WJ, Sprung CL. The ACCP-SCCM consensus conference on sepsis and organ failure. Chest 1992; 101: 1481-1483, doi: 10.1378/chest.101.6.1481.

2. Nascimento DC, Alves-Filho JC, Sonego F, Fukada SY, Pereira MS, Benjamim C, et al. Role of regulatory $\mathrm{T}$ cells in long-term immune dysfunction associated with severe sepsis. Crit Care Med 2010; 38: 1718-1725, doi: 10.1097/ CCM.0b013e3181e78ad0.

3. Benjamim CF, Hogaboam CM, Lukacs NW, Kunkel SL. Septic mice are susceptible to pulmonary aspergillosis. Am J Pathol 2003; 163: 2605-2617, doi: 10.1016/S0002-9440 (10)63615-2.

4. Otto GP, Sossdorf M, Claus RA, Rodel J, Menge K, Reinhart $\mathrm{K}$, et al. The late phase of sepsis is characterized by an increased microbiological burden and death rate. Crit Care 2011; 15: R183, doi: 10.1186/cc10332.

5. Boomer JS, To K, Chang KC, Takasu O, Osborne DF, Walton $\mathrm{AH}$, et al. Immunosuppression in patients who die of sepsis and multiple organ failure. JAMA 2011; 306: 25942605, doi: 10.1001/jama.2011.1829.

6. Salomao R, Martins PS, Brunialti MK, Fernandes ML, Martos LS, Mendes ME, et al. TLR signaling pathway in patients with sepsis. Shock 2008; 30 (Suppl 1): 73-77, doi: 10.1097/SHK.0b013e318181af2a.

7. Salomao R, Brunialti MK, Rapozo MM, Baggio-Zappia GL, Galanos C, Freudenberg M. Bacterial sensing, cell signaling, and modulation of the immune response during sepsis. Shock 2012; 38: 227-242, doi: 10.1097/SHK.0b013e318 262c4b0.

8. Janeway CA Jr, Medzhitov R. Innate immune recognition. Annu Rev Immunol 2002; 20: 197-216, doi: 10.1146/annurev. immunol.20.083001.084359.

9. Ishii KJ, Koyama S, Nakagawa A, Coban C, Akira S. Host innate immune receptors and beyond: making sense of microbial infections. Cell Host Microbe 2008; 3: 352-363, doi: 10.1016/j.chom.2008.05.003.

10. Pugin J, Heumann ID, Tomasz A, Kravchenko VV, Akamatsu $Y$, Nishijima M, et al. CD14 is a pattern recognition receptor. significantly lower after 7 days of therapy compared to admission. Interestingly, expression of the TLR2, TLR4 and CXCR2 receptors on neutrophils at follow-up was differentially modulated in survivors and nonsurvivors. Expression of TLR2 and TLR4 on the surface of neutrophils was diminished while expression of CXCR2 was augmented at follow-up for survivors and remained stable for nonsurvivors. These results may reflect cellular reprogramming, a mechanism that is possibly involved in decreasing inflammation and preserving infection control. The lack of modulation of TLR2 and TLR4 expression on the monocytes of septic patients compared to healthy volunteers highlights the important role of the intracellular mechanisms of inflammatory control.

\section{Acknowledgments}

Research supported by FAPESP (\#2006/58744-1).

Immunity 1994; 1: 509-516, doi: 10.1016/1074-7613(94) 90093-0.

11. Landmann R, Muller B, Zimmerli W. CD14, new aspects of ligand and signal diversity. Microbes Infect 2000; 2: 295304, doi: 10.1016/S1286-4579(00)00298-7.

12. Ley K. Molecular mechanisms of leukocyte recruitment in the inflammatory process. Cardiovasc Res 1996; 32: 733742, doi: 10.1016/0008-6363(96)00066-1.

13. Rose JJ, Foley JF, Murphy PM, Venkatesan S. On the mechanism and significance of ligand-induced internalization of human neutrophil chemokine receptors CXCR1 and CXCR2. J Biol Chem 2004; 279: 24372-24386, doi: 10 . 1074/jbc.M401364200.

14. Skubitz KM, Campbell KD, Skubitz AP. CD66a, CD66b, CD66c, and CD66d each independently stimulate neutrophils. J Leukoc Biol 1996; 60: 106-117.

15. Martins PS, Brunialti MK, Martos LS, Machado FR, Assunçao MS, Blecher S, et al. Expression of cell surface receptors and oxidative metabolism modulation in the clinical continuum of sepsis. Crit Care 2008; 12: R25, doi: 10.1186/cc6801.

16. Brunialti MK, Martins PS, Barbosa de Carvalho $\mathrm{H}$, Machado FR, Barbosa LM, Salomao R. TLR2, TLR4, CD14, CD11B, and $C D 11 C$ expressions on monocytes surface and cytokine production in patients with sepsis, severe sepsis, and septic shock. Shock 2006; 25: 351-357, doi: 10.1097| 01.shk.0000217815.57727.29.

17. Aalto $\mathrm{H}$, Takala $\mathrm{A}$, Kautiainen $\mathrm{H}$, Siitonen $\mathrm{S}$, Repo $\mathrm{H}$. Monocyte CD14 and soluble CD14 in predicting mortality of patients with severe community acquired infection. Scand $J$ Infect Dis 2007; 39: 596-603, doi: 10.1080/003655407011 99808.

18. Calvano JE, Agnese DM, Um JY, Goshima M, Singhal R, Coyle SM, et al. Modulation of the lipopolysaccharide receptor complex (CD14, TLR4, MD-2) and toll-like receptor 2 in systemic inflammatory response syndrome-positive patients with and without infection: relationship to tolerance. Shock 2003; 20: 415-419, doi: 10.1097/01.shk.0000092269. 
01859.44 .

19. Tansho-Nagakawa S, Ubagai T, Kikuchi-Ueda T, Koshio O, Koshibu Y, Kikuchi H, et al. Analysis of membrane antigens on neutrophils from patients with sepsis. $J$ Infect Chemother 2012; 18: 646-651, doi: 10.1007/s10156-012-0386-7.

20. Jorgensen PF, Wang JE, Almlof M, Thiemermann C, Foster SJ, Solberg R, et al. Peptidoglycan and lipoteichoic acid modify monocyte phenotype in human whole blood. Clin Diagn Lab Immunol 2001; 8: 515-521.

21. Gomes NE, Brunialti MK, Mendes ME, Freudenberg M, Galanos C, Salomão R. Lipopolysaccharide-induced expression of cell surface receptors and cell activation of neutrophils and monocytes in whole human blood. Braz $J$ Med Biol Res 2010; 43: 853-858, doi: 10.1590/S0100879X2010007500078.

22. Muller Kobold AC, Tulleken JE, Zijlstra JG, Sluiter W, Hermans J, Kallenberg CG, et al. Leukocyte activation in sepsis; correlations with disease state and mortality. Intensive Care Med 2000; 26: 883-892, doi: 10.1007/s0013 40051277.

23. Harter L, Mica L, Stocker R, Trentz O, Keel M. Increased expression of toll-like receptor-2 and -4 on leukocytes from patients with sepsis. Shock 2004; 22: 403-409, doi: 10. 1097/01.shk.0000142256.23382.5d.

24. Schaaf B, Luitjens K, Goldmann T, van Bremen T, Sayk F, Dodt $C$, et al. Mortality in human sepsis is associated with downregulation of Toll-like receptor 2 and CD14 expression on blood monocytes. Diagn Pathol 2009; 4: 12, doi: 10. 1186/1746-1596-4-12.

25. Vijay-Kumar M, Sanders CJ, Taylor RT, Kumar A, Aitken JD, Sitaraman SV, et al. Deletion of TLR5 results in spontaneous colitis in mice. J Clin Invest 2007; 117: 39093921.

26. Hemmi H, Takeuchi O, Kawai T, Kaisho T, Sato S, Sanjo H, et al. A Toll-like receptor recognizes bacterial DNA. Nature 2000; 408: 740-745, doi: 10.1038/35047123.

27. Plitas G, Burt BM, Nguyen HM, Bamboat ZM, DeMatteo RP. Toll-like receptor 9 inhibition reduces mortality in polymicrobial sepsis. J Exp Med 2008; 205: 1277-1283, doi: 10. 1084/jem.20080162.

28. Trevelin SC, Alves-Filho JC, Sonego F, Turato W, Nascimento DC, Souto FO, et al. Toll-like receptor 9 activation in neutrophils impairs chemotaxis and reduces sepsis outcome. Crit Care Med 2012; 40: 2631-2637, doi: 10.1097/CCM.0b013e318258fb70.

29. Martins PS, Kallas EG, Neto MC, Dalboni MA, Blecher S, Salomao R. Upregulation of reactive oxygen species generation and phagocytosis, and increased apoptosis in human neutrophils during severe sepsis and septic shock. Shock 2003; 20: 208-212, doi: 10.1097/01.shk.0000079425. 52617.db.

30. Santos SS, Brunialti MK, Rigato O, Machado FR, Silva E,
Salomao R. Generation of nitric oxide and reactive oxygen species by neutrophils and monocytes from septic patients and association with outcomes. Shock 2012; 38: 18-23, doi: 10.1097/SHK.0b013e318257114e.

31. Brunialti MK, Santos MC, Rigato O, Machado FR, Silva E, Salomao R. Increased percentages of $\mathrm{T}$ helper cells producing IL-17 and monocytes expressing markers of alternative activation in patients with sepsis. PLoS One 2012; 7: e37393, doi: 10.1371/journal.pone.0037393.

32. Salomao R, Brunialti MK, Gomes NE, Mendes ME, Diaz $\mathrm{RS}$, Komninakis $\mathrm{S}$, et al. Toll-like receptor pathway signaling is differently regulated in neutrophils and peripheral mononuclear cells of patients with sepsis, severe sepsis, and septic shock. Crit Care Med 2009; 37: 132-139, doi: 10. 1097/CCM.0b013e318192fbaf.

33. Foster SL, Hargreaves DC, Medzhitov R. Gene-specific control of inflammation by TLR-induced chromatin modifications. Nature 2007; 447: 972-978.

34. Mendes ME, Baggio-Zappia GL, Brunialti MK, Fernandes ML, Rapozo MM, Salomao R. Differential expression of toll-like receptor signaling cascades in LPS-tolerant human peripheral blood mononuclear cells. Immunobiology 2011; 216: 285-295, doi: 10.1016/j.imbio.2010.07.008.

35. Rochon YP, Kavanagh TJ, Harlan JM. Analysis of integrin (CD11b/CD18) movement during neutrophil adhesion and migration on endothelial cells. J Microsc 2000; 197: 15-24, doi: 10.1046/j.1365-2818.2000.00645.x.

36. Lin RY, Astiz ME, Saxon JC, Rackow EC. Altered leukocyte immunophenotypes in septic shock. Studies of HLA-DR, CD11b, CD14, and IL-2R expression. Chest 1993; 104: 847-853, doi: 10.1378/chest.104.3.847.

37. Marsik C, Mayr F, Cardona F, Derhaschnig U, Wagner OF, Jilma B. Endotoxaemia modulates Toll-like receptors on leucocytes in humans. Br J Haematol 2003; 121: 653-656, doi: 10.1046/j.1365-2141.2003.04350.x.

38. Cummings CJ, Martin TR, Frevert CW, Quan JM, Wong VA, Mongovin SM, et al. Expression and function of the chemokine receptors CXCR1 and CXCR2 in sepsis. $J$ Immunol 1999; 162: 2341-2346.

39. Juffermans NP, Dekkers PE, Peppelenbosch MP, Speelman P, van Deventer SJ, van der Poll T. Expression of the chemokine receptors CXCR1 and CXCR2 on granulocytes in human endotoxemia and tuberculosis: involvement of the p38 mitogen-activated protein kinase pathway. J Infect Dis 2000; 182: 888-894, doi: 10.1086/ 315750.

40. Rios-Santos F, Alves-Filho JC, Souto FO, Spiller F, Freitas $A$, Lotufo CM, et al. Down-regulation of CXCR2 on neutrophils in severe sepsis is mediated by inducible nitric oxide synthase-derived nitric oxide. Am J Respir Crit Care Med 2007; 175: 490-497, doi: 10.1164/rccm.2006011030C. 